\title{
An open randomized active-controlled clinical trial with low-dose SKA cytokines versus DMARDs evaluating low disease activity
} maintenance in patients with rheumatoid arthritis

\author{
This article was published in the following Dove Press journal: \\ Drug Design, Development and Therapy \\ 29 March 2017 \\ Number of times this article has been viewed
}

\section{LS Martin-Martin \\ F Giovannangeli ${ }^{2}$ \\ E Bizzi ${ }^{2}$ \\ U Massafra ${ }^{2}$ \\ E Ballanti ${ }^{2}$ \\ $\mathrm{MCassol}^{3}$}

A Migliore ${ }^{2}$

'Department of Internal Medicine, Regina Apostolorum Hospital, ${ }^{2}$ Operative Unit of Rheumatology, ${ }^{3}$ Department of Internal Medicine, San Pietro Fatebenefratelli Hospital, Rome, Italy
Correspondence: E Bizzi

Operative Unit of Rheumatology, San Pietro Fatebenefratelli Hospital,

Via Cassia 600, Rome 00189, Italy

Tel +390633585802

Email bizzi.emanuele@gmail.com
Background: Biologic agents are currently the strongest immunosuppressive drugs able to induce remission in rheumatoid arthritis (RA). One of the objectives of the medical scientific community now is how to maintain remission or low disease activity (LDA). The aim of this trial is to evaluate the contribution of low-dose sequential kinetic activation (SKA) IL-4, IL-10, and anti-IL-1 antibodies $(10 \mathrm{fg} / \mathrm{mL})$ in patients affected by RA in maintaining LDA or remission obtained after biological therapy.

Method: This is a randomized, open, active-controlled, prospective, Phase IV trial. Disease activity score (DAS28), clinical disease activity index, simplified disease activity index, erythrocyte sedimentation rate and C-reactive protein levels, global health assessment, and pain visual analog scale were evaluated at baseline visit and then every 3 months together with an assessment of side effects till 12 months. Thirty-nine RA patients were enrolled and randomized to continue disease-modifying antirheumatic drugs (DMARDs) therapy or to receive a combination of SKA low-dose cytokines formulated in concentration of $10 \mathrm{fg} / \mathrm{mL}$ orally administered at a dose of 20 drops/d for 12 consecutive months.

Results: The rate of maintenance of LDA at 12 months was superior in the group treated with low-dose cytokines compared with patients treated with DMARDs, $66.7 \%$ and $42.1 \%$, respectively; however, the difference between the groups was not statistically significant. No side effects were reported in both groups.

Conclusion: This is the first study using a combination of three low-dose cytokines in RA, after data published on psoriasis. These data suggest that the use of a combination of low-dose SKA cytokines may be an opportunity to explore in the management of RA.

Keywords: RA, low-dose cytokines, low-dose antibodies, SKA, biological agents, low disease activity

\section{Introduction}

Rheumatoid arthritis (RA) is an autoimmune inflammatory disease that can potentially lead to disability; in fact, about $30 \%$ of patients affected by RA are no longer able to perform a job after 3 years of disease. ${ }^{1}$ The antitumor necrosis factor- $\alpha$ (TNF- $\alpha$ ) agents, called "biological agents", represent the most effective therapy available for patients with RA. ${ }^{2}$ Biologic drugs are currently the strongest immunosuppressive drugs able to induce remission in RA, as they inhibit the proinflammatory cytokines further expressed during the acute phase of illness. However, their economic cost is high, and the biological risk of prolonged treatment remains uncertain. Preliminary evidence of 
loss of efficacy over time is emerging, and therefore rotation between the available biological drugs is often necessary to maintain a good clinical response. ${ }^{3}$ Another unsolved problem is to know how long the patient should take the biologic medications; in addition, in the early stages, once the remission of the disease is obtained, it is unclear whether it is correct to suspend the biological therapy. Biologic drugs are now used as second-line drug in case of failure of conventional therapy with Methotrexate, A Cyclosporin, or Leflunomide, considered as first-line therapy and ientified as disease-modifying antirheumatic drugs (DMARDs). ${ }^{4}$

One of the purposes of the medical scientific community now is to get not only a subjective and objective improvement of the symptomatic relief, but also the remission of the disease and the achievement of the lowest level of activity (low disease activity, LDA). ${ }^{5}$

In this context, identifying the therapeutic strategies that may maintain remission or the LDA either with therapy with DMARDs (first line) or with the biological (second line) is the aim of the scientific community. In addition to the two options of continuing treatment with DMARDs or biologic drugs, after achieving remission or LDA, with the related economic costs and safety issues, there is a third option of biological regulation with low doses of anti-inflammatory cytokines regulators.

At the present state of knowledge, there are numerous data on both the murine and human models suggesting that it might be possible to intervene directly in the inflammatory process of RA through the use of certain cytokines and inhibitors able to modulate at least in part the complex network of cytokines involved in the pathogenesis of RA. ${ }^{6,7}$ The contemporary use of more than one biologic drug at standard dosage is actually impossible, as such a combination would be burdened by severe side effects. ${ }^{8}$ On the contrary, low doses $(10 \mathrm{fg} / \mathrm{mL})$ of cytokines, already in use in Italy, in the United States, and in several other countries for various diseases, may allow their simultaneous use with superior efficacy to their single use without causing serious adverse effect. This could allow a more physiological, though slower, modulation of the pathological process. ${ }^{9} \mathrm{RA}$ is an immunemediated disease with an imbalance of the immune system Th1/Th2 in favor of the Th1 clone, with cytokines TNF- $\alpha$, Interleukin (IL)-6, IL-1 increase, and reduction of IL-4 and IL-10. ${ }^{10,11}$ In this condition, the combined use of low doses of IL-4, IL-10, and anti-IL-1 antibodies may contribute to maintaining the balance of the immune system.

In addition, according to studies on animals and human beings, the particular process of sequential kinetic activation
(SKA) allows us to amplify the therapeutic effects without adverse effects. ${ }^{12,13}$

Gariboldi et $\mathrm{al}^{14}$ recently published a study in which oral administration of low-dose IL-12 plus Interferon- $\gamma$ is able to quench the bronchial hyperresponsiveness in mice. The antiasthma effect was confirmed by histological analysis of the lungs and by bronchoalveolar lavage.

The aim of this trial is to evaluate whether the use of lowdose SKA IL-4, IL-10, and anti-IL-1 antibodies (10 fg/mL) in patients affected by RA can maintain LDA or remission obtained after biological therapy or DMARDs, with a treatment and observation period of 12 months.

\section{Materials and methods Study design}

This is a randomized, open, two arms, active-controlled, prospective, Phase IV trial to evaluate the maintenance of LDA or remission with the use of low-dose SKA IL-4, IL-10, and anti-IL-1 antibodies $(10 \mathrm{fg} / \mathrm{mL})$. The treatment and observation period was 12 months (Figure 1).

Inclusion criteria for patient enrollment:

- RA diagnosed according to ACR criteria $^{15}$

- Duration of disease $<3$ years

- Disease activity score of 28 joints (DAS28) $<3.2$ after biologic drugs and/or DMARD therapy

- Patients who have reached the state of remission or LDA after treatment with biologic drugs (Adalimumab, Etanercept, Infliximab, Certolizumab pegol, Golimumab) or after one therapy with DMARDs

- Patients able to adhere to the procedures of the study and fill out questionnaires

- Written informed consent received from each participants. Exclusion criteria were the following:

- Age $<18$ years

- Disease duration $>3$ years

- Presence of comorbidities that would contraindicate therapy with biological agents or immunosuppressant drugs (infection in progress, or a history of tuberculosis or cancer).

The study was conducted in accordance with the principles of the Declaration of Helsinki (Seoul 2008) and Good Clinical Practice guidelines (CPMP/ICH/135/95), and the study and the consent procedures were approved by the Ethics Committee of the San Pietro Fatebenefratelli Hospital, Rome. The trial was registered with EudraCT number 2011003016-23 through the database of the National Clinical Trials Monitoring Center Database (Osservatorio, OsSC) of the Italian Medicines Agency. 


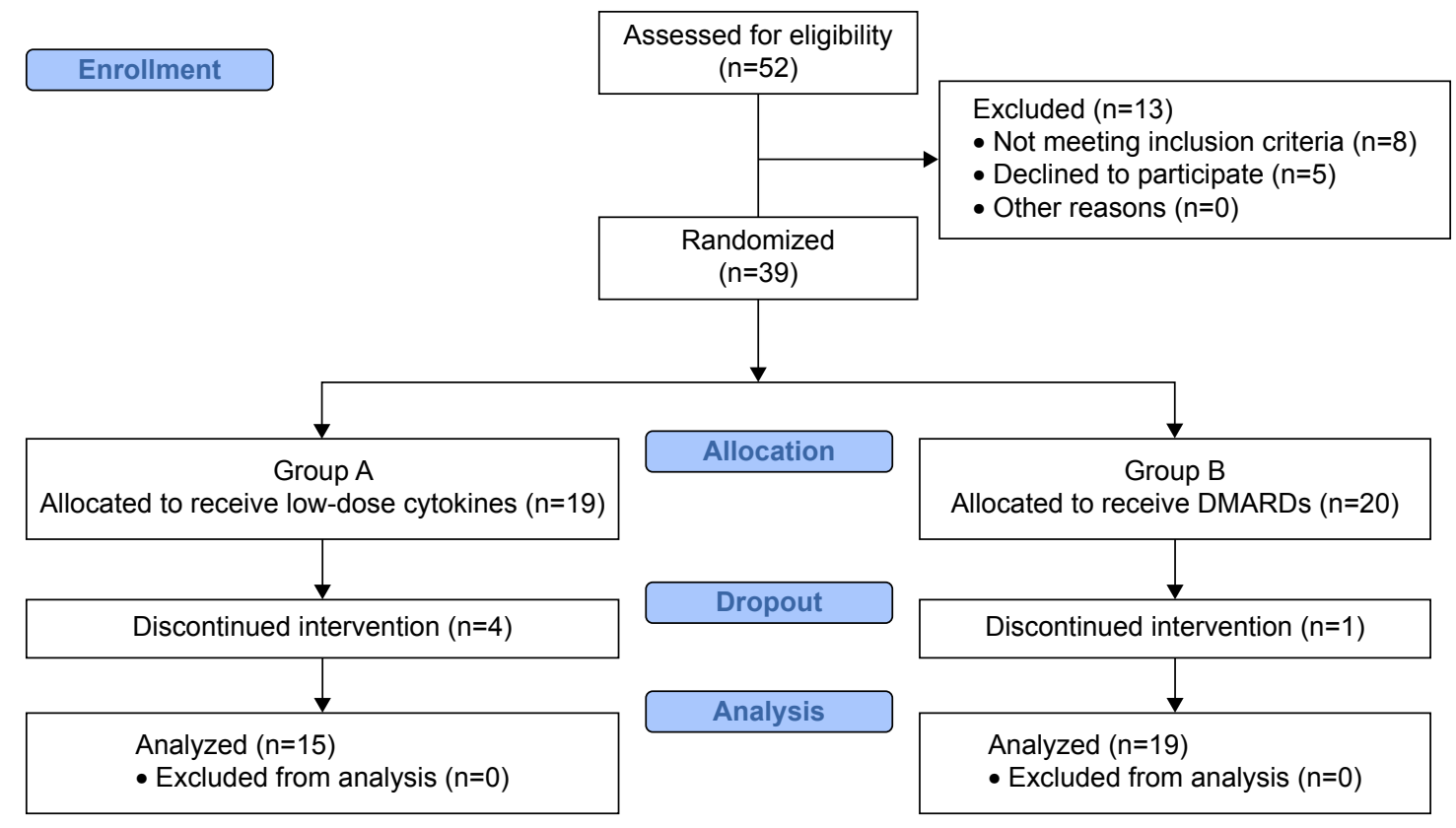

Figure I Flowchart diagram reporting study enrollment, allocation, dropouts distribution, and patients included for analysis. Abbreviation: DMARDs, disease-modifying antirheumatic drugs.

\section{Study procedure}

Subjects were enrolled at the Rheumatology Unit, San Pietro Hospital Fatebenefratelli, Rome, between July 2011 and March 2014. A total of 52 subjects were screened and 39 were enrolled and randomized as shown in the clinical trial flow diagram (Figure 1). In detail, five males and 34 females (mean age: 55.19) with RA, diagnosed according to ACR criteria, were enrolled in the study. At the screening visit, the following actions were performed: verification of criteria for inclusion and exclusion in the study, collection of demographic characteristics and complete anamnesis and signature of written informed consent was received from each subject for this study. At baseline visit (T0), clinical parameters such as DAS28, clinical disease activity index (CDAI), simplified disease activity index (SDAI), swollen and tender joints, global health assessment (GHA), and pain visual analog scale (VAS) were evaluated, and laboratory testing such as erythrocyte sedimentation rate (ESR), rheumatoid factor (RF), and C-reactive protein (CRP) was performed. Such parameters were then evaluated every 3 months together with an assessment of side effects. In addition, the use of NSAIDs, whose use was permitted (Celecoxib), was assessed at every control visit.

\section{Outcomes}

The primary endpoint of this study is to assess whether and how long the association of low-dose SKA IL-4, IL-10, and anti-IL-1 antibodies $(10 \mathrm{fg} / \mathrm{mL})$ can maintain LDA obtained by previous treatment with anti-TNF- $\alpha$ biologic agents and/or DMARDs by the evaluation of activity parameters such as DAS28, CDAI, and SDAI.

As secondary endpoints we also evaluated at baseline and every 3 months pain VAS rating on a $0-10$ scale, GHA, erythrosedimentation rate (ESR, using the Westergren method), CRP levels, and NSAIDs consumption, and we aimed to identify the eventual predictors of the effectiveness of the association of low-dose SKA IL-4, IL-10, anti-IL-1 antibodies $(10 \mathrm{fg} / \mathrm{mL})$ in the maintenance of remission obtained with biological drugs. We also performed an overall safety evaluation of drugs administered in the two arms.

\section{Treatments}

SKA-cytokines/antibodies were prepared by GUNA Laboratories (GUNA S.p.a., Milan, Italy) using the standardized method. ${ }^{13}$ IL-4, IL-10, and anti-IL-1 antibodies were activated by sequential serial dilution (1:100) in 30\% hydroalcoholic solution and kinetically energized by a shaking procedure (vertical shaking; $10 \mathrm{~cm}$ motion range; shaking speed corresponding to 100 oscillations in 10 seconds). Subjects who satisfied the eligibility criteria were randomly allocated to Group A (Cytokines arm) or Group B (DMARDs arm), and identified by their initials and a serial number.

Group A is made up of patients who achieved an LDA or remission of the disease after 6-8 months of biological therapy or conventional therapy with DMARDs, after withdrawing biologic drugs or conventional therapy, and were 
treated with Guna-IL-4, Guna-IL-10, and Guna-Anti-IL-1 formulated at a concentration of $10 \mathrm{fg} / \mathrm{mL}$ SKA. Each preparation was orally administered at a dose of $20 \mathrm{drops} / \mathrm{d}$ for 12 consecutive months. The use of NSAID (Celecoxib $200 \mathrm{mg} / \mathrm{d}$ ) has been permitted on request. Group B is made up of patients who achieved LDA or remission of the disease after 6-8 months of biological therapy or conventional therapy with DMARDs that will continue with conventional therapy (DMARDs). The use of NSAID (Celecoxib $200 \mathrm{mg} / \mathrm{d}$ ) has been permitted on request. Patients in Group A were permitted to continue DMARDs-based therapy.

\section{Randomization}

Subjects who satisfied the eligibility criteria were randomized using software with a 60 random list for the two groups ( $A$ and $B$ ) in a 1:1 ratio. Block randomization was performed with a block size of 4 . The subjects were assigned to Group A or B, according to the sequential order of enrollment.

\section{Statistical analysis}

A descriptive analysis was performed for all the demographic variables and clinical features at baseline. Student's $t$-test for paired data was conducted to evaluate the clinical efficacy parameters (DAS28) of treatment at 12 months, compared to baseline. In case of violation of the assumptions underlying the aforementioned parametric statistical tests, the analysis was performed with a nonparametric method, in particular, the Wilcoxon test. The McNemar test was used in each group of patients to evaluate changes observed at different times for qualitative variables.

Laboratory data were analyzed using descriptive statistics (mean, standard error, minimum, and maximum) at each time of detection for assessment of tolerability. Changes in laboratory parameters between baseline and the time T12 were assessed using a parametric method (Student's $t$-test for dependent samples) or, in the case of breach of commitments, a nonparametric method (the Wilcoxon test). The retention of patients in the state of LDA in each group was evaluated by the Kaplan-Meyer b curve.

\section{Results}

Fifty-two subjects underwent screening. Thirty-nine of these were enrolled. Five patients did not complete the study (Figure 1, Table 1). As for the main characteristics of patients at baseline, no differences were observed between the two groups, except for mean age, which turned out to be slightly lower in Group A.
Table I Demographic and clinical characteristics of patients included in the study at TO

\begin{tabular}{llll}
\hline & Group A & & Group B \\
\cline { 2 - 2 } & Mean \pm SD & & Mean \pm SD \\
\hline Subjects $(\mathrm{n})$ & 15 & 19 \\
Age & $47.08 \pm \mathrm{II} . \mathrm{II}$ & & $63.3 \pm \mathrm{II} .48$ \\
Females (\%) & 92.85 & & $85.7 \mathrm{I}$ \\
Disease duration (years) & $3.58 \pm \mathrm{I} .62$ & & $2.56 \pm 0.82$ \\
Previous DMARDs therapy $(\mathrm{n})$ & $2 / 15(13.3 \%)$ & & $3 / 19(15.8 \%)$ \\
Previous biologic therapy $(\mathrm{n})$ & $13 / 15(86.7 \%)$ & $16 / 19(84.2 \%)$ \\
DAS28 & 2.62 & 2.46 \\
CDAl & 6.14 & 2.80 \\
SDAl & 4.99 & 2.23 \\
Swollen joints & 0.50 & 0.14 \\
Tender joints & 1.33 & 1.14 \\
GHA & 21.56 & 16.50 \\
ESR (mm/h) & 13.67 & 14.26 \\
CRP (mg/dL) & 0.70 & 2.18 \\
RF & 50.85 & 83.78 \\
VAS (0-I0) & 3.38 & 3.08 \\
\hline
\end{tabular}

Notes: Group A: patients achieving remission or LDA undergoing low dose cytokines therapy. Group B: patients achieving remission or LDA undergoing conventional DMARDs therapy.

Abbreviations: T0, baseline visit; DMARDs, disease-modifying antirheumatic drugs; DAS, disease activity score; CDAl, clinical disease activity index; LDA, low disease activity; SDAI, simplified disease activity index; GHA, global health assessment; ESR, erythrocyte sedimentation rate; CRP, C-reactive protein; RF, rheumatoid factor; VAS, visual analog scale; SD, standard deviation.

\section{Primary endpoint}

The maintenance of LDA at 12 months is obtained in $66.7 \%$ of subjects treated with low-dose cytokines (Group A; $n=10$ ) and in $42.1 \%$ of patients treated with DMARDs (Group B; $\mathrm{n}=8$ ); the difference between the groups is not statistically significant (Fisher's exact test: $P=0.185$ )

In Group A, 2 subjects were treated at the same time with DMARDs (Methotrexate) and low-dose cytokines.

\section{DAS28}

DAS28 values are similar in the two groups at baseline (Mann-Whitney $U$-test: $P=0.3991$ ) as well as at 12 months (Mann-Whitney $U$-test: $P=0.1030)$. Group A maintains constant values of DAS28 (Friedman test: $P=0.41604$ ), while in Group B, DAS28 values are on the rise (Friedman test: $P=0.00198$ ), with a significant difference (test according to Conover: $P<0.05)$ between T0 and T9, T0 and T12, T3 and T9, and T3 and T12 (Figure 2).

\section{CDAI}

CDAI scores are similar in the two groups at baseline (MannWhitney $U$-test: $P=0.7317$ ) as well as at 12 months (MannWhitney $U$-test: $P=0.0510)$. Group A shows a constant sealing over time (Friedman test: $P=0.84645$ ), while values 


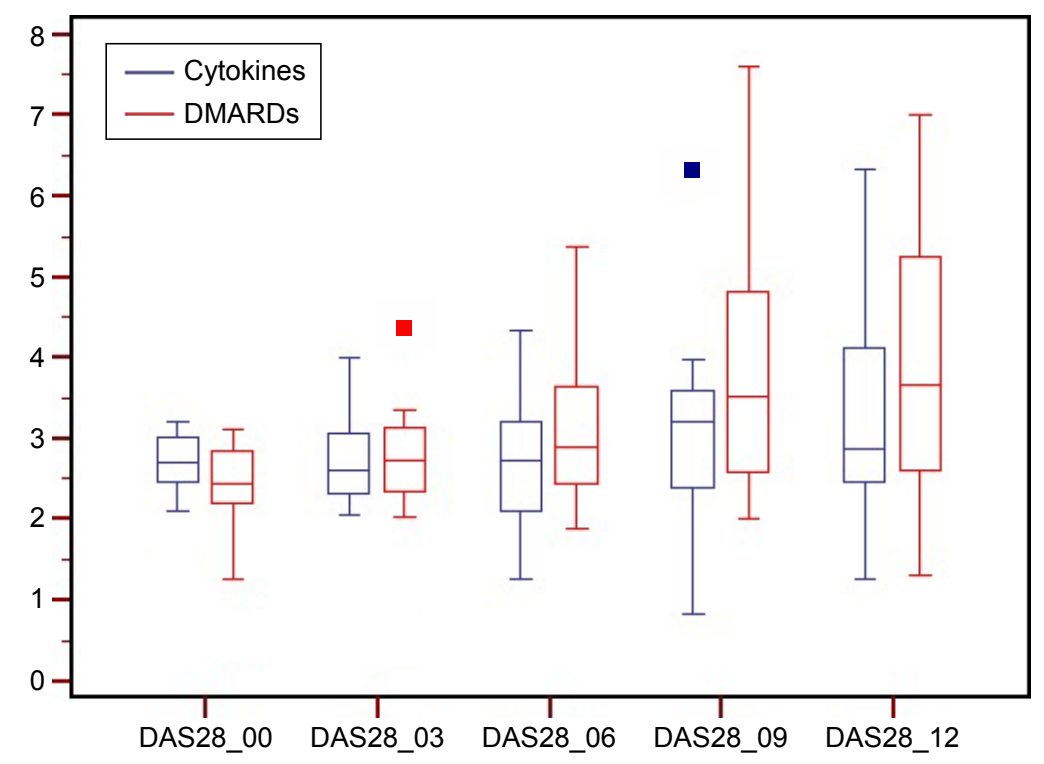

Figure 2 DAS28 in patients receiving low-dose cytokines or conventional therapy as evaluated at baseline or every 3 months. Note: Squares report mean value and standard deviation of DAS28 score at various time points.

Abbreviations: DAS, disease activity score; DMARDs, disease-modifying antirheumatic drugs.

are on the rise in Group B (Friedman test: $P=0.00004)$, with a significant difference (test according to Conover: $P<0.05$ ) between $\mathrm{T} 0$ and T6, T0 and T9, T0 and T12, T3 and T9, T3 and T12, T6 and T9, and T6 and T12 (Figure 3).

\section{SDAI}

The SDAI showed no statistical difference between the two groups at baseline (Mann-Whitney $U$-test: $P=0.9223$ ) as well as at 12 months (Mann-Whitney $U$-test: $P=0.0790$ ). Group A showed a constant intragroup sealing (Friedman test: $P=0.56774)$, while a significant intragroup difference was shown in Group B (Friedman test: $P<0.00001$ and test according to Conover: $P<0.05)$ between the following time points: T0 and T6, T9 and T0, T0 and T12, T3 and T9, T12 and T3, T6 and T9, and T6 and T12 (Figure 4).

\section{Secondary endpoint}

\section{Pain VAS}

The pain VAS values are similar between the two groups at both baseline visit (Mann-Whitney $U$-test: $P=0.7336$ ) and

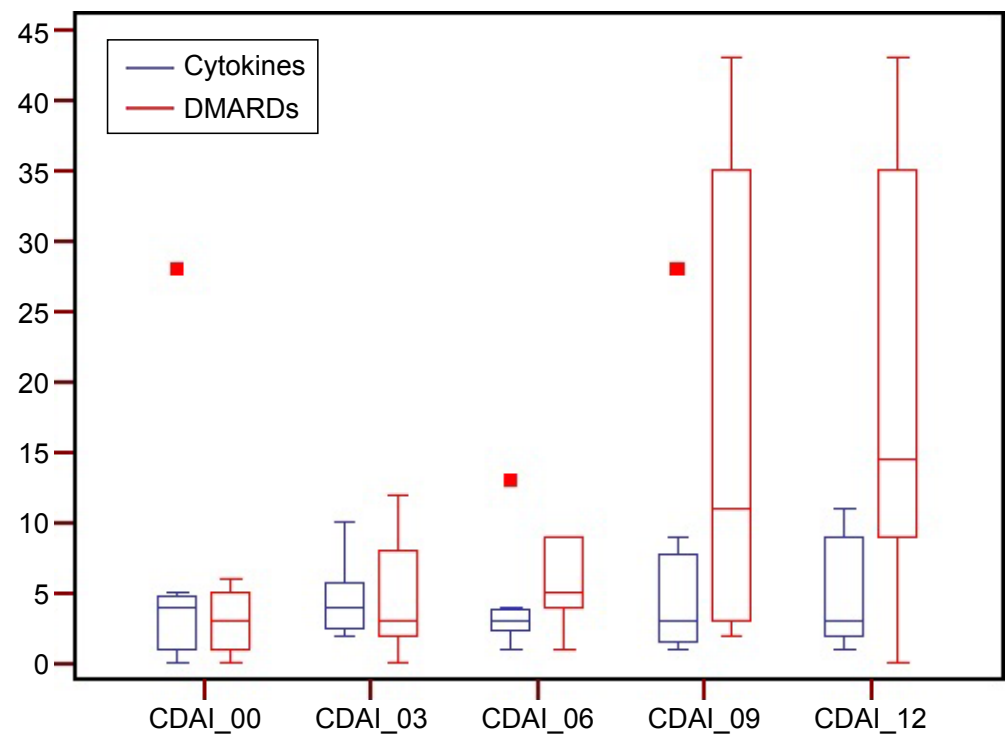

Figure $3 \mathrm{CDAl}$ in patients receiving low-dose cytokines or conventional therapy as evaluated at baseline or every 3 months. Note: Squares report mean value and standard deviation of CDAl score at various time points.

Abbreviations: CDAl, clinical disease activity index; DMARDs, disease-modifying antirheumatic drugs. 


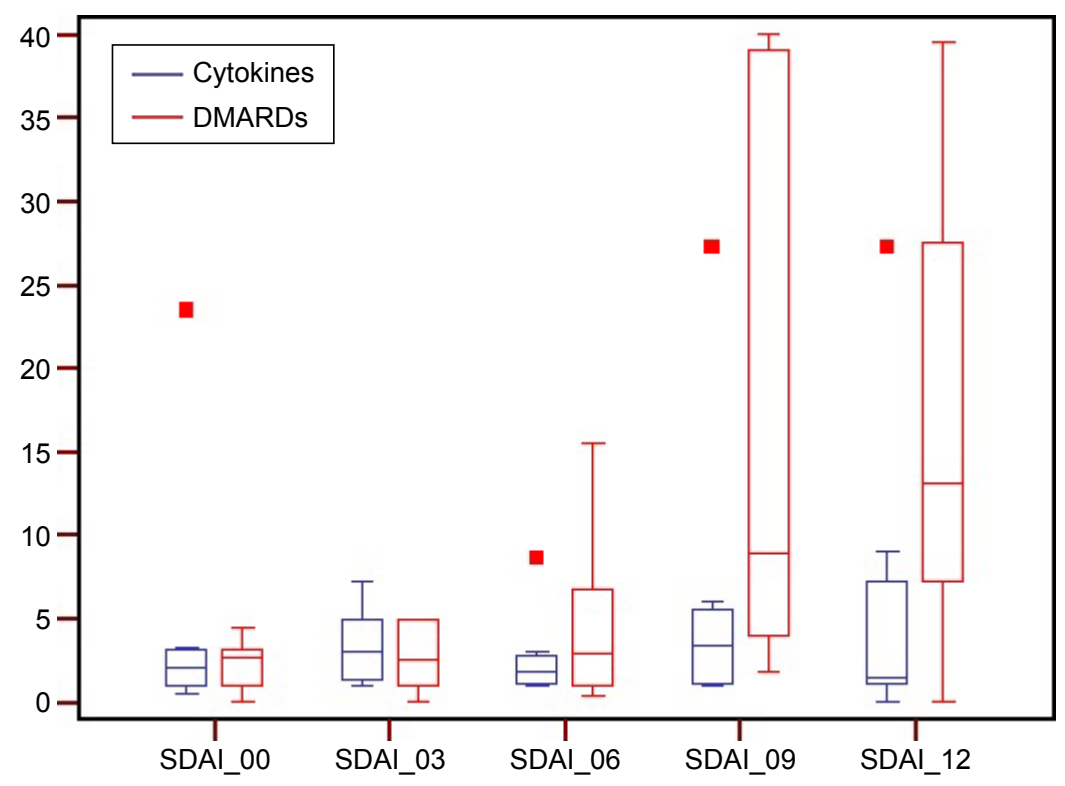

Figure 4 SDAI in patients receiving low-dose cytokines or conventional therapy as evaluated at baseline or every 3 months. Note: Squares report mean value and standard deviation of SDAl score at various time points.

Abbreviations: SDAI, simple disease activity index; DMARDs, disease-modifying antirheumatic drugs.

12 months follow-up (Mann-Whitney $U$-test: $P=0.1772$ ). Patients maintain constant levels without any intragroup difference as shown by the Friedman test; $P$-values were, respectively, 0.79490 in Group A and 0.12474 in Group B (Figure 5).

\section{GHA}

GHA values did not show any statistical difference between the two groups at baseline (Mann-Whitney $U$-test: $P=0.4998$ ) and at 12 months (Mann-Whitney $U$-test: $P=0.3269$ ). Patients maintain constant values in both groups: Friedman test: $P=0.19770$ in Group A and Friedman test: $P=0.05608$ in Group B (Figure 6).

\section{ESR}

ESR mean values did not show any significant intergroup difference at baseline (Mann-Whitney $U$-test: $P=0.7153$ ) as well as at 12 months (Mann-Whitney $U$-test: $P=0.0699$ ). Similarly, no intragroup significant differences were reported; Friedman test $P$-values were, respectively, 0.53603 in Group A and 0.08022 in Group B (Figure 7).

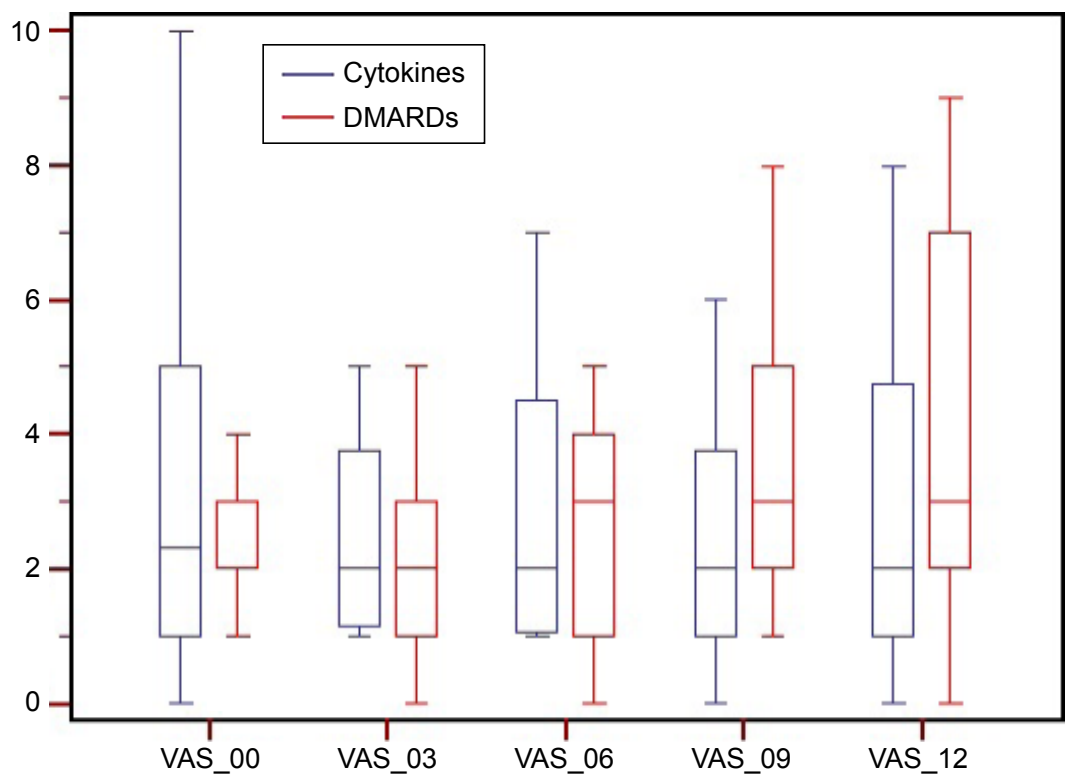

Figure 5 VAS in patients receiving low-dose cytokines or conventional therapy as evaluated at baseline or every 3 months. Abbreviations: VAS, visual activity score on a scale I-I0; DMARDs, disease-modifying antirheumatic drugs. 


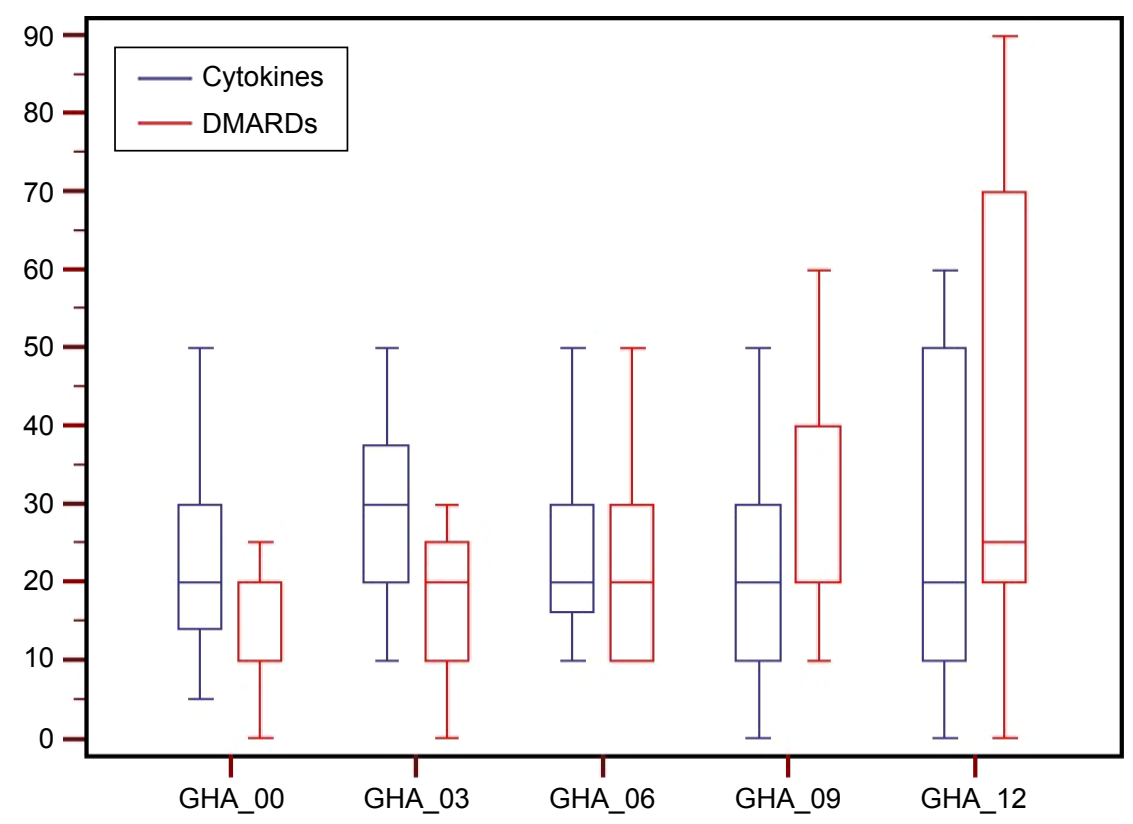

Figure 6 GHA in patients receiving low-dose cytokines or conventional therapy as evaluated at baseline or every 3 months. Abbreviations: GHA, global health assessment; DMARDs, disease-modifying antirheumatic drugs.

\section{CRP}

The CRP mean values are lower in Group A at baseline (Mann-Whitney $U$-test: $P=0.0078$ ), but similar at 12 months without any significant statistical difference (Mann-Whitney $U$-test: $P=0.0966)$. Patients show intragroup constant levels; Friedman test was, respectively, $P=0.69002$ in Group A and $P=0.22356$ in Group B (Figure 8).

\section{NSAIDs (Celecoxib 200 mg/die) consumption evaluation}

No differences were observed in the consumption of Celecoxib between two groups at any time point, although the small sample size, owing to the small percentage of patients needing to take this medication, does not allow certain conclusions to be drawn.

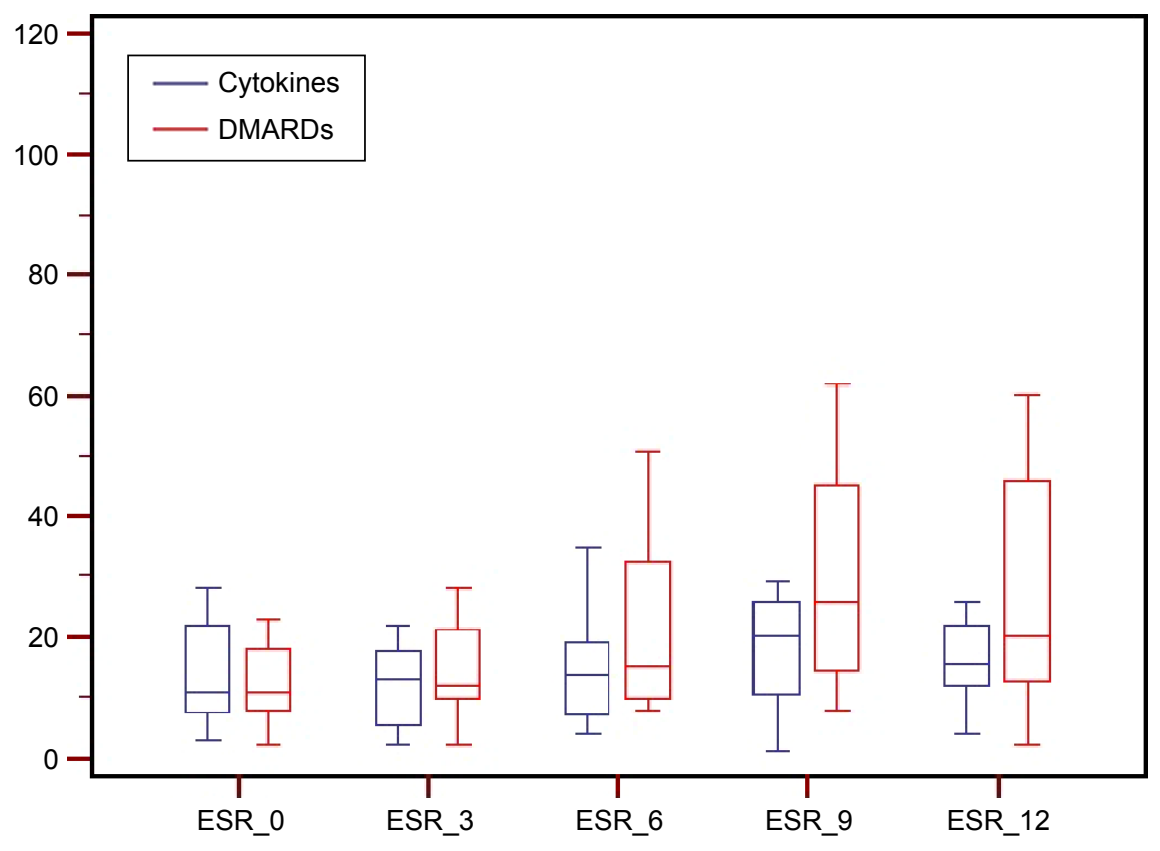

Figure 7 ESR in patients receiving low-dose cytokines or conventional therapy as evaluated at baseline or every 3 months. Abbreviations: ESR, erythrosedimentation rate; DMARDs, disease-modifying antirheumatic drugs. 


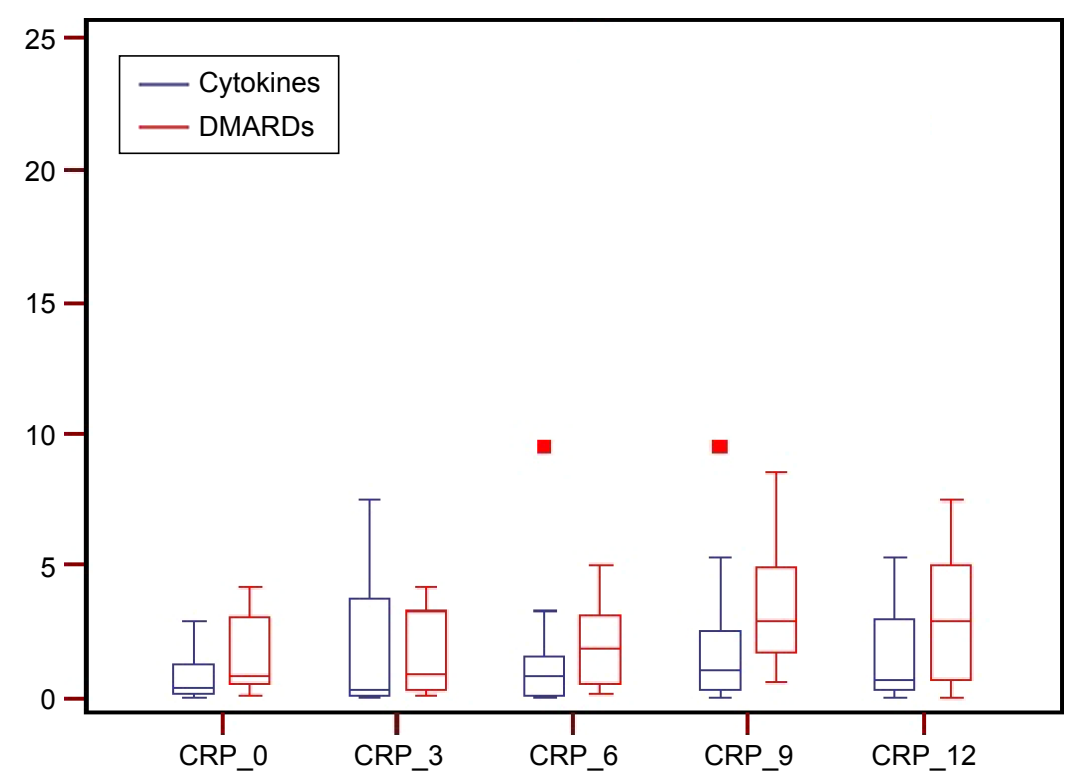

Figure $8 \mathrm{CRP}$ in patients receiving low-dose cytokines or conventional therapy as evaluated at baseline or every 3 months. Note: Squares report mean value and standard deviation of CRP score at various time points.

Abbreviations: CRP, C reactive protein; DMARDs, disease-modifying antirheumatic drugs.

\section{Predictors of the effectiveness of cytokines} association therapy in the maintenance of remission

Covariance analysis regarding the impact of the condition of LDA or remission in the results of DAS28 showed no statistically significant differences (Friedman test: $P=0.530$ ). Because of the small sample size, no statistical analysis was performed as for CDAI and SDAI.

\section{Safety assessment}

A total of 34 patients were exposed to treatments during 12 months. Respectively, 15 subjects in Group A, composed of patients who achieved an LDA or remission of the disease after biological therapy or conventional therapy with DMARDs, were treated with Guna-IL-4, Guna-IL-10, and Guna-Anti-IL-1 formulated concentration $10 \mathrm{fg} / \mathrm{mL}$, and 19 patients in the Group B, who similarly achieved an LDA or remission of the disease after biological therapy or conventional therapy with DMARDs, were treated with conventional therapy.

No adverse events were reported by investigators in both groups.

\section{Discussion}

This is the first study using low-dose cytokine in RA, after those published on psoriasis. In fact, Roberti et al ${ }^{9}$ studied the pharmacological activity and safety of three low-dose cytokines - Guna-IL-4, Guna-IL-10, and Guna-IL-11 - at a concentration of $10 \mathrm{fg} / \mathrm{mL}$ in patients affected by moderateto-slight psoriasis vulgaris in a double-blind, randomized, placebo-controlled clinical trial involving 48 patients. They reported a psoriasis area severity index significant reduction in the 41 evaluated patients.

In this study, the maintenance of LDA or remission at 12 months is obtained, respectively, in $66.7 \%$ of subjects treated with low-dose cytokines (Group A) and in $42.1 \%$ of subjects treated with DMARDs (Group B).

Even though the results do not reach the primary endpoint, it is important to note that they cannot be considered conclusive as evaluated on a sample not sufficiently powerful (power is much lower than $80 \%$ because of the low number of subjects). However, there are sufficient reasons to believe that treatment with cytokines gives a better result in respect to the standard DMARDs-based therapy, considering the trend of the experimental arm.

Values of DAS28, CDAI, and SDAI seem to show a common behavior of the variables, eg, even if the sample was too small to detect intergroup differences, the nonparametric ANOVA (Friedman test) shows that substantially stable values of the variables have been maintained in Group A, whereas a significant increase is observed for Group B. More precisely, running a pairwise analysis (with Conover's test adjusted for multiple comparisons), we notice that increases, compared to baseline, begin to be significant $(P<0.05)$ at T9 for DAS28, at T6 for CDAI, and even at T6 for SDAI. Moreover, in Group A, values are kept at a lower degree of dispersion, as reported looking at box plots. Such differences were not observed in ESR and CRP levels, and these data are difficult to interpret, especially after having observed 
different trends in the two groups for all other observed indicators.

Taken together, these data suggest the opportunity to use low-dose SKA cytokine in RA. In particular, this study seems to suggest that the differences between the two groups emerge in the sixth month of treatment after the beginning of remission, when the group treated with low-dose SKA cytokines keeps the requirements of remission in a homogeneous manner, while the group treated with DMARDs gets progressively worse with a wide range of intragroup variability. The data seem to confirm the hypothesis that therapy with low-dose SKA cytokines might induce a progressive regulation and a stabilization of the immune system that may justify the smaller dispersion of the sample at least in a subset of patients. As for the differences in terms of results obtained in the study on psoriasis in respect to the results obtained in this study, it is difficult to explain why this kind of therapy may be more effective in a pathology such as psoriasis, but again the small number of patients included in this study as well as the deep pathogenic differences between psoriasis and RA may explain this dissimilarity.

Although further studies are needed, these preliminary data allow us to hypothesize that one out of four patients could benefit from the low-dose SKA treatment to maintain remission. These data suggest that protocols need to be developed aimed at identifying predictors of response.

That difference in maintaining the low levels of DAS28 can be estimated as $25 \%$, since low level is actually verified at $\mathrm{T} 12$ in 10 of 15 patients $(67 \%)$ and in 8 of 19 patients (42\%).

From the perspective of clinical practice, therapy with low-dose SKA cytokines should be considered among the tools for the management of disease remission obtained with biological agents in RA patients, as well as for dermatologists in vitiligo management. ${ }^{16}$ The availability of a systemic treatment based on the oral administration of low-dose activated signaling molecules represents an opportunity for the specialists to overcome some specific pitfalls of currently available therapeutic protocols. It responds to the need for new strategies for the treatment of RA with biological agents, or the target of cytokines combination, as well as contributing to the evidence on the issue of management of remission in RA. Although several drugs with different mechanisms of action are available for the treatment of RA, a significant percentage of patients show an inadequate response to biological treatment. It can be hypothesized that treatment failure could be due to the multifactorial nature of the disease or to the existence of several subsets of disease.
Consequently, new research approaches have to be introduced with the aim of gaining the target of clinical response in all RA patients. Taking inspiration from the oncologic or cardiologic fields, we could hypothesize a new regimen of treatment with combined low-dose or ultralow-dose biological agents to avoid important side effects but acting simultaneously on different targets, since literature data of a combination of biological agents administered at the registered dose are not safe. ${ }^{17-21}$ These data confirm the safety profile of these molecules, even in combination, as reported in other studies, and in addition, this is the first study to show data for a continuous period of 12 months. ${ }^{8}$

The low cost of the low-dose SKA cytokines is another factor to be taken into account for the possibility of extending the treatments to a greater number of patients, considering also the optimal safety profile.

This clinical trial presents some limitations. First, the number of patients is not sufficient to establish statistically significant differences, and a larger study population is needed to increase the certainty of results. Even if follow-up times are enough to show a different trend between the groups, a longer follow-up could give us more information about the duration on LDA in the low-dose cytokine group. Finally, the study did not compare treatments with a placebo arm.

However, these data are consistent with the trend toward a personalization of treatment in RA patients, according to current international literature and good clinical practice.

\section{Conclusion}

In conclusion, the use of low-dose SKA cytokines/antibodies (IL-4, IL-10, and anti-IL-1) simultaneously administered orally has shown a good efficacy in RA patients in maintaining LDA at least in a subset of patients who have undergone remission after biological drugs or conventional therapy. The safety profile has been confirmed excellent since no adverse event was reported in any of the RA patients treated. The availability of low-dose products containing diluted and SKA-activated cytokines represents a new opportunity to explore for the global management of RA patients also in the era of biological agents.

\section{Acknowledgments}

We thank Vincenzo Miranda (Clinical Research Unit Guna S.p.a Milan-Italy) for his helpful scientific support. We also thank Michele Nichelatti for the statistical analysis, Marilena Campanella (Clinical Research Unit Guna S.p.a Milan-Italy) for her technical support in data management, and Federica Melzi for her linguistic assistance. 


\section{Disclosure}

The authors report no conflicts of interest in this work.

\section{References}

1. Lee DM, Weinblatt ME. Rheumatoid arthritis. Lancet. 2001;358(9285): 903-911.

2. Alonso-Ruiz A, Pijoan JI, Ansuategui E, Urkaregi A, Calabozo M, Quintana A. Tumor necrosis factor alpha drugs in rheumatoid arthritis: systematic review and metaanalysis of efficacy and safety. $B M C$ Musculoskelet Disord. 2008;9:52.

3. Strehblow C, Haberhauer G, Fasching P. Comparison of different biologic agents in patients with rheumatoid arthritis after failure of the first biologic therapy. Wien Med Wochenschr. 2010;160(9-10):225-229.

4. Demoruelle MK, Deane KD. Treatment strategies in early rheumatoid arthritis and prevention of rheumatoid arthritis. Curr Rheumatol Rep. 2012;14(5):472-480.

5. van der Helm-van Mil AH, Breedveld FC, Huizinga TW. Aspects of early arthritis. Definition of disease activity states in early arthritis: remission versus minimal disease activity. Arthritis Res Ther. 2006;8:216.

6. Burska A, Boissinot M, Ponchel F. Cytokines as biomarkers in rheumatoid arthritis. Mediators Inflamm. 2014;2014:545493.

7. Venkatesha SH, Dudics S, Acharya B, Moudgil KD. Cytokine-modulating strategies and newer cytokine targets for arthritis therapy. Int J Mol Sci. 2015;16(1):887-906.

8. Migliore A, Ballanti E, Laganà B, Martin LS, Frediani B. Biologic agents for rheumatoid arthritis: can we hypothesize new strategies of treatment? Med Hypotheses. 2014;82(1):117-121.

9. Roberti ML, Ricottini L, Capponi A, et al. Immunomodulating treatment with low dose interleukin-4, interleukin-10 and interleukin-11 in psoriasis vulgaris. J Biol Regul Homeost Agents. 2014;28(1):133-139.

10. Schulze-Koops H, Kalden JR. The balance of Th1/Th2 cytokines in rheumatoid arthritis. Best Pract Res Clin Rheumatol. 2001;15(5): 677-691.

11. Roberts CA, Dickinson AK, Taams LS. The interplay between monocytes/macrophages and CD4(+) $\mathrm{T}$ cell subsets in rheumatoid arthritis. Front Immunol. 2015;6:571.
12. Radice E, Bellone G, Miranda V. Enhancement of the immunostimulatory functions of ex vivo-generated dendritic cells from early-stage colon cancer patients by consecutive exposure to low doses of sequentialkinetic-activated IL-4 and IL-12. A preliminary study. Transl Oncol. 2015;8(4):327-338.

13. Radice E, Miranda V, Bellone G. Low-doses of sequential-kineticactivated interferon- $\gamma$ enhance the ex vivo cytotoxicity of peripheral blood natural killer cells from patients with early-stage colorectal cancer. A preliminary study. Int Immunopharmacol. 2014;19(1):66-73.

14. Gariboldi S, Palazzo M, Zanobbio L, et al. Low dose oral administration of cytokines for treatment of allergic asthma. Pulm Pharmacol Ther. 2009;22(6):497-510.

15. Aletaha D, Neogi T, Silman AJ, et al. 2010 rheumatoid arthritis classification criteria: an American College of Rheumatology/European League Against Rheumatism collaborative initiative. Ann Rheum Dis. 2010;69(9):1580-1588.

16. Lotti T, Hercogova J, Fabrizi G. Advances in the treatment options for vitiligo: activated low-dose cytokines-based therapy. Expert Opin Pharmacother. 2015;16(16):2485-2496.

17. Zwerina J, Hayer S, Tohidast-Akrad M, et al. Single and combined inhibition of tumor necrosis factor, interleukin-1, and RANKL pathways in tumor necrosis factor-induced Arthritis. Arthritis Rheum. 2004;50:277-290.

18. Alzabin S, Abraham SM, Taher TE, et al. Incomplete response of inflammatory arthritis to TNFa blockade is associated with the Th17 pathway. Ann Rheum Dis. 2012;71:1741-1748.

19. Genovese MC, Cohen S, Moreland L, et al. Combination therapy with etanercept and anakinra in the treatment of patients with rheumatoid arthritis who have been treated unsuccessfully with methotrexate. Arthritis Rheum. 2004;50:1412-1419.

20. Weinblatt M, Combe B, Covucci A, et al. Safety of the selective costimulation modulator abatacept in rheumatoid arthritis patients receiving background biologic and nonbiologic disease-modifying antirheumatic drugs: a one-year randomized, placebo-controlled study. Arthritis Rheum. 2006;54:2807-2816.

21. Greenwald MW, Shergy WJ, Kaine JL, et al. Evaluation of the safety of rituximab in combination with a tumor necrosis factor inhibitor and methotrexate in patients with active rheumatoid arthritis: results from a randomized controlled trial. Arthritis Rheum. 2011;63(3):622-632.
Drug Design, Development and Therapy

\section{Publish your work in this journal}

Drug Design, Development and Therapy is an international, peerreviewed open-access journal that spans the spectrum of drug design and development through to clinical applications. Clinical outcomes, patient safety, and programs for the development and effective, safe, and sustained use of medicines are the features of the journal, which

\section{Dovepress}

has also been accepted for indexing on PubMed Central. The manuscript management system is completely online and includes a very quick and fair peer-review system, which is all easy to use. Visit http://www.dovepress.com/testimonials.php to read real quotes from published authors. 